“C 2019 IEEE. Personal use of this material is permitted. Permission from IEEE must be obtained for all other uses, in any current or future media, including reprinting/republishing this material for advertising or promotional purposes, creating new collective works, for resale or redistribution to servers or lists, or reuse of any copyrighted component of this work in other works." 


\title{
Tomography of Quantum Dots in a Non-Hermitian Photonic Chip
}

\author{
Simon J. U. White ${ }^{1,2}$, Kai Wang ${ }^{3,2}$, Toan Trong Tran ${ }^{1}$, Mehran Kianinia ${ }^{1}$, James Titchener ${ }^{3,4}$, Markus \\ Gräfe $^{5}$, Sarah Fischbach ${ }^{6}$, Sven Rodt ${ }^{6}$, Jin-Dong Song ${ }^{7}$, Stephan Reitzenstein ${ }^{6}$, Igor Aharonovich ${ }^{1}$, \\ Andrey A. Sukhorukov ${ }^{3}$, Alexander Szameit ${ }^{2}$ and Alexander S. Solntsev ${ }^{1}$ \\ 1. School of Mathematical and Physical Sciences, Faculty of Science, University of Technology Sydney, Ultimo, NSW, 2007, Australia \\ 2. Institut für Physik, Universität Rostock, Albert-Einstein-Straße 23, 18059 Rostock, Germany \\ 3. Nonlinear Physics Centre, Research School of Physics and Engineering, The Australian National University, Canberra, ACT, 2601, \\ Australia \\ 4. Quantum Technology Enterprise Centre, Quantum Engineering Technology Labs, H. H. Wills Physics Laboratory and Department of \\ Electrical and Electronic Engineering, University of Bristol, Tyndall Avenue, Bristol, BS8 1FD, United Kingdom \\ 5. Fraunhofer Institute for Applied Optics and Precision Engineering, Albert-Einstein-Straße 7, 07745 Jena, Germany \\ 6. Institut für Festkrperphysik, Quantum Devices Group, Technische Universität Berlin, Hardenbergstraße 36, 10623 Berlin, Germany \\ 7. Center for Opto-Electronic Convergence Systems, Korea Institute of Science and Technology, Seoul 136-791, Korea
}

Quantum optical information systems offer the potential for secure communication and fast quantum computation. To fully characterise a quantum optical system one has to use quantum tomography [1]. Integration of quantum optics onto photonic chips provides advantages such as miniaturisation and stability, and also significantly improves quantum tomography using both re-configurable [2], and more recently, simpler static designs [3,4]. These on-chip designs have, so far, only used probabilistic single photon sources. Here we are working towards quantum tomography using a true deterministic source - a quantum dot. The scheme of the proposed experiment is shown in Fig. 1A. So far we have fabricated and characterised the performance of an InGaAs quantum dot monolithically integrated into a microlens [5], and completed the design, fabrication and classical characterisation of a photonic chip for quantum tomography.

The chip operates by splitting a state of a photon, generated by a quantum dot, from two inputs ( 0 and 1$)$ into four outputs. This is achieved through a combination of lossless and non-Hermitian couplers (couplers with loss). Non-Hermitian couplers typically lead to poor performance of quantum optical systems, but here we show that by carefully controlling the loss we can optimise the chip for tomography. We represent a photon quantum state as a point on a sphere, known as a Bloch sphere. Since we use 4 output ports, any photon state can be represented by a combination of the four output intensities. To optimise the chip, we ensure that all outputs are spread over the entire sphere, see Fig. 1B. This decreases the susceptibility to error, known as the condition number. After classical characterisation [6] at $890 \mathrm{~nm}$, the condition number [7] for this chip was found to be around 4, which is over 2 times lower than conventional tomography techniques. Currently we are working on a complete quantum dot tomography experiment.
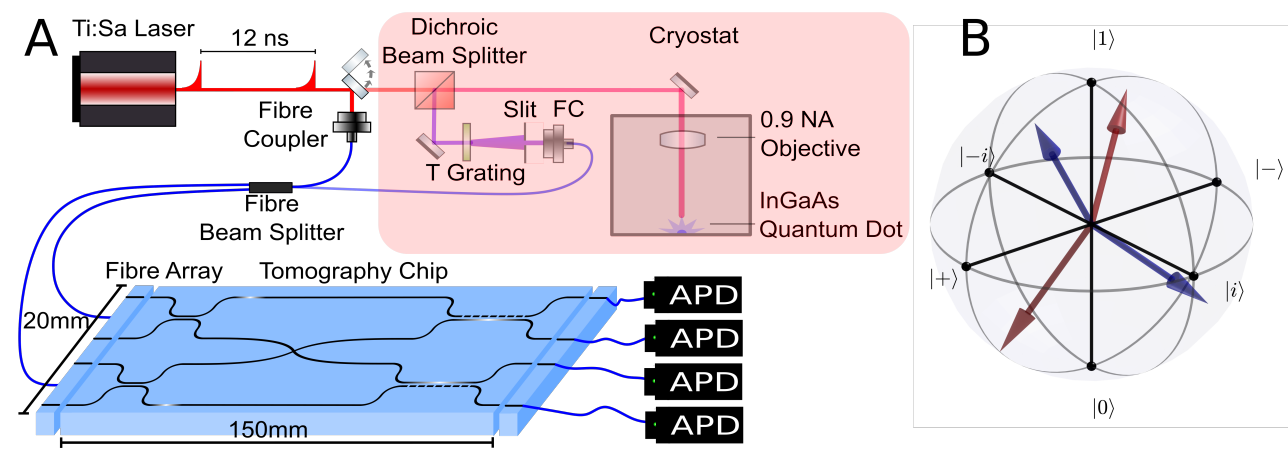

Fig. 1 A. The schematic of the quantum dot tomography experiment. A Ti:Sapphire laser can be coupled into a tomography chip for classical characterisation (completed), or into a quantum dot (red shaded area, currently in progress). B. Experimentally determined projections of the four outputs of the tomography chip onto the Bloch sphere.

[1] D. F. James, P. G. Kwiat, W. J. Munro, and A. G. White, “Measurement of qubits,” Phys. Rev. A 64, 052312 (2001).

[2] P. J. Shadbolt, M. R. Verde, A. Peruzzo, A. Politi, A. Laing, M. Lobino, J. C. F. Matthews, M. G. Thompson, and J. L. OBrien, “Generating, manipulating and measuring entanglement and mixture with a reconfigurable photonic circuit," Nat. Photon. 6, 45 (2011).

[3] J. G. Titchener, M. Gräfe, R. Heilmann, A. S. Solntsev, A. Szameit, and A. A. Sukhorukov, "Scalable on-chip quantum state tomography," npj Quantum Inf. 4, 19 (2018)

[4] K. Wang, J. G. Titchener, S. S. Kruk, L. Xu, H.-P. Chung, M. Parry, I. I. Kravchenko, Y.-H. Chen, A. S. Solntsev, Y. S. Kivshar, D. N. Neshev, and A. A. Sukhorukov, "Quantum metasurface for multiphoton interference and state reconstruction," Science, 361(6407), 1104 LP-1108 (2018).

[5] M. Gschrey, A. Thoma, P. Schnauber, M. Seifried, R. Schmidt, B. Wohlfeil, L. Krger, J.-H. Schulze, T. Heindel, S. Burger, F. Schmidt, A. Strittmatter, S. Rodt, and S. Reitzenstein, "Highly indistinguishable photons from deterministic quantum-dot microlenses utilizing threedimensional in situ electron-beam lithography," Nat. Commun. 6, 7662 (2015).

[6] R. Heilmann, M. Gräfe, S. Nolte, and A. Szameit, "A novel integrated quantum circuit for high-order W-state generationand its highly precise characterization," Sci. Bull. 60, 96100 (2015).

[7] A. Miranowicz, K. Bartkiewicz, J. Peina, M. Koashi, N. Imoto, and F. Nori, "Optimal two-qubit tomography based on local and global measurements: Maximal robustness against errors as described by condition numbers,” Phys. Rev. A 90, 062123 (2014). 